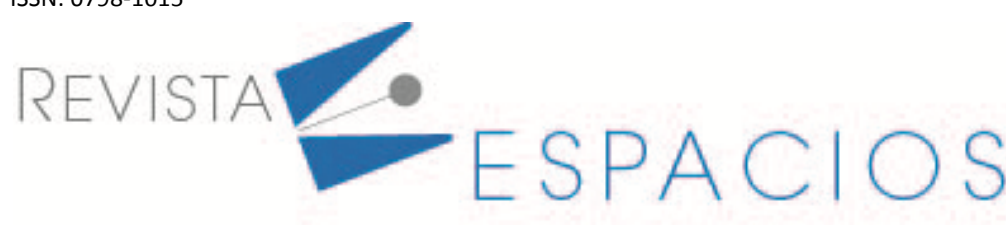

\title{
Estilos de aprendizaje en la UCEVA: una caracterización de estudiantes de Licenciatura en Lenguas Extranjeras
}

\section{Learning Styles at UCEVA: a characterization of undergraduate students in Foreign Languages}

ROMERO MARTÍNEZ, Gonzalo ${ }^{1}$

SANDOVAL, Edgar de J. ${ }^{2}$

SUÁREZ DÁVILA, Marco F. ${ }^{3}$

LASSO CARDONA, Luis A. ${ }^{4}$

\begin{abstract}
Resumen
En Colombia, los resultados de las pruebas Saber y PISA no han arrojado resultados satisfactorios en áreas de lectura y el uso del inglés. La investigación tiene como objetivo identificar los estilos de aprendizaje y el perfil predominante de los estudiantes de Licenciatura en Lenguas Extranjeras con Énfasis en Inglés de la Unidad Central del Valle del Cauca en Tuluá, Colombia. Se identificó que el estilo predominante fue el Reflexivo. En este contexto, los docentes deben incentivar el proceso de aprendizaje mediante actividades pedagógicas innovadoras que lleven al estudiante a responder al porqué de las situaciones del mundo real, con el objetivo de llegar a conclusiones más profundas de los temas analizados.

Palabras clave: estilos de aprendizaje, CHAEA, enseñanza.

Abstract

In Colombia, the results of the Saber and PISA tests have not yielded satisfactory results in the areas of reading and the use of English. The objective of the research is to identify the learning styles and the predominant profile of the students of the Bachelor of Foreign Languages at Unidad Central del Valle del Cauca in Tuluá, Colombia. It was identified that the predominant style was Reflective. In this context, teachers must encourage the learning process through innovative pedagogical activities that make students answer the why of the real situations in order to conclude deeply about analyzed topics.

key words: styles of learning, CHAEA, learning.
\end{abstract}

\section{Introducción}

En un estudio realizado por Alonso, Gallego y Honey (2007) se evidenció que cada alumno tiene diferentes maneras de aprendizaje relacionadas a la forma en que este percibe, procesa y obtiene conocimientos, es decir, que existen diferentes Estilos de Aprendizaje (EA).

\footnotetext{
1 Unidad Central del Valle del Cauca, Colombia. Profesor tiempo completo, Facultad de Educación. MSc Bilingual Education. gromero@uceva.edu.co

2 Unidad Central del Valle del Cauca, Colombia. Profesor tiempo completo, Facultad de Ingenierías. MSc Educación y TIC. esandoval@uceva.edu.co

${ }^{3}$ Unidad Central del Valle del Cauca, Colombia. Profesor catedrático, Facultad de Educación. Msc en Didáctica del Ingles. msuarez@uceva.edu.co

${ }^{4}$ Universidad del Valle, Colombia. Profesor Asistente, Facultad de Ingeniería. MSc. Gestión de la Tec. Educación. luis.lasso@correounivalle.edu.co
} 
Una de las principales necesidades que han presentado los estudiantes al momento de aprender es encontrar una manera de hacerlo acorde a sus carencias. A esta manera de aprender en el área pedagógica, se le denomina Estilo, el cual es definido por Alonso, Gallego y Honey (1994) como un término que se usa para señalar una serie de comportamientos distintos reunidos bajo un solo nombre, y si es referido al aprendizaje ofrece indicadores que ayudan a guiar las interacciones de la persona con la realidad favoreciendo la comprensión y los hábitos de lectura.

Si bien es cierto, diferentes investigadores han abordado el tema de EA, este estudio se centrará en la conceptualización que hacen Honey y Mumford (1986), quienes replantean las teorías Kolb en 1974, definiendo cuatro etapas: experiencia, reflexión, elaboración de hipótesis y aplicación. Cada una de las etapas se asocia a los EA: Reflexivo, Activo, Teórico y Pragmático, y explican que los estilos son el conjunto de elementos psicológicos, aspectos cognitivos, afectivos y fisiológicos que tienden a manifestarse integralmente una vez el individuo tenga que afrontar o abordar un contexto de aprendizaje.

Los rasgos cognitivos dan cuenta de la manera en que los alumnos organizan las temáticas, forman y usan conceptualizaciones, comprenden e interpretan la información, solucionan problemas, escogen y organizan medios de representación (visual, auditivo, kinestésico, etc). El componente afectivo se relaciona con los aspectos motivacionales internos o externos que presenta el estudiante y las expectativas, deseos e inquietudes que tienen que ver con el aprendizaje, y consecuentemente las características fisiológicas se relacionan con el biorritmo y biotipo del estudiante.

Merece atención mencionar que el tema de EA es afín con las estrategias de Enseñanza-Aprendizaje, las cuales para Monereo $(2002 ; 2007)$ son un proceso de toma de decisiones intencionales, las cuales son seleccionadas por el estudiante, para llevar de forma ordenada, los aprendizajes, procedimientos y actitudes para cumplir con un objetivo específico, teniendo en cuenta los contextos educativos en donde sucede la acción. La finalidad esencial de las estrategias en cualquier acto de aprendizaje es proporcionar los mejores medios tangibles o intangibles para la comprensión de las temáticas que surgen del exterior al sistema cognitivo del estudiante, a través de este procedimiento se ve necesaria la organización y observación de los datos que ingresan, su posterior clasificación, categorización, recuperación, almacenamiento y salida de la información. Cuando un estudiante sigue o responde estas etapas, se puede decir que muestra un comportamiento estratégico para abordar su proceso de aprendizaje (Monereo, 2007; De la Fuente y Justicia, 2003).

En relación a los EA anteriormente mencionados y la dependencia de estos en los procesos de lectura, numerosos autores han manifestado la necesidad de indagar sobre las estrategias pedagógicas que intervienen en la lectura de una lengua extranjera desde la comprensión de esta como una compleja relación entre el sujeto que lee, el texto y el autor (Cassany, 2004; Grabe y Stoller, 2002; Quintana et al., 2019).

De acuerdo con Solé (2012) los sistemas de representación de la lengua escrita, permiten pensar, comunicar y aprender, por tanto, son consecuencia de dilatados procesos que se generan y desarrollan en el seno de actividades sociales: en la familia, en la escuela y en los diversos contextos, a los que progresivamente se van incorporando los niños y jóvenes, lo que conlleva a que se genere una identidad propia en un individuo que establecerá vínculos en una sociedad. Lo anterior se ve plasmado en la investigación de Loayza (2017), que determinó que existe una relación entre los EA y la comprensión lectora en estudiantes de 4to. Grado, al establecer una correlación entre variables con la prueba estadística Rho de Spearman. Lo que permitió establecer que, en este grado de formación de básica primaria, el niño, aún se encuentra delimitando y construyendo su EA, pero que paulatinamente se vuelven mucho más metódicos en el momento de centrar su interés. De igual forma, se comprobó la existencia de diversos EA y la importancia de ellos en el proceso de enseñanza que condiciona el aprendizaje. Por su parte, Paul y Elder (2005), establecieron la relación entre el desarrollo del pensamiento, el aprendizaje y la educación, donde el pensamiento es evaluado constantemente en su estructura 
básica con el fin de mejorarse. Por ello, la educación debe plantearse qué hacer para poder aportar a la construcción del conocimiento en los estudiantes, de tal forma que la memorización no sea la única estrategia para el aprendizaje.

En Colombia, históricamente los resultados de las pruebas locales como ICFES Saber, aplicada a los grados 3우, 5으, 90 y 11으, que evalúa competencias básicas en áreas como: Lectura crítica, ciencias naturales, ciencias sociales, matemáticas e inglés, e internacionales como PISA (Programa Internacional para la Evaluación de Estudiantes) sobre el nivel de lectura, no han sido muy alentadoras, ni para español como lengua materna, ni de inglés como lengua extranjera (Sánchez-Jabba, 2013), posiblemente a consecuencia de los bajos niveles de lectura en el país, que lo ubican muy por debajo del promedio regional y mundial, y que según estadísticas de la Encuesta de Cultura (EC) realizada por el Departamento Administrativo Nacional de Estadística (DANE) y el Centro Regional para el Fomento del Libro en América Latina y el Caribe (CERLALC), el 61,3\% de la población de 12 a 25 años fue la que más leyó libros en los últimos 12 meses. No obstante, esa cifra tiende a disminuir, pues en 2010 ese mismo promedio estaba en $68,5 \%$. Por su parte, tan solo el $44,1 \%$ de las personas entre los 26 y 40 años, y el 40,2\% entre los 41 a 64 años tienen hábitos de lectura (CERLALC, 2012; El Espectador, 2014).

A lo anterior hay que agregarle que en la mayoría de los casos la enseñanza del segundo idioma no es una prioridad en la educación básica primaria y secundaria, con lo cual se desperdician esos años de adquisición del idioma, lo que lleva a bajos niveles generalizados, que posteriormente implica transformaciones de fondo en el currículo que permitan conocer y desarrollar los EA de cada estudiante para hacer más efectivo su proceso académico, y lograr llevarlos a niveles más desarrollados de lectura y proeficiencia en inglés (El Tiempo, 2018).

Debido a lo anterior, una de las medidas tomadas por las instituciones educativas de Colombia, que buscan realizar "un cambio drástico en el currículum educativo" a través de sus distintos programas en los tres niveles: primaria, bachillerato y universidad, es el fortalecimiento del bilingüismo, específicamente en inglés, siendo esta una de las tareas que debe enmarcarse con el diseño de un currículo adecuado para desarrollar y afianzar el proceso de Enseñanza-Aprendizaje, lo que requiere de la incorporación de un componente esencial de carácter curricular y metodológico que tenga en cuenta escenarios de EA para concebir estrategias con miras a lograr mejores resultados y que pongan al país en una posición competitiva a nivel regional y luego mundial (El País, 2013).

La presente investigación tiene como objetivo identificar los EA y el perfil predominante de los estudiantes de I y II semestre de la Licenciatura en Lenguas Extranjeras con énfasis en Inglés de la Unidad Central del Valle del Cauca (UCEVA) por medio de la aplicación del CHAEA, puesto que en los últimos años se ha identificado una problemática en el proceso de comprensión lectora, en ambos idiomas; inglés y español. El estudio se dividió básicamente en 3 fases: 1) recolección de datos para la construcción del marco teórico, 2) aplicación y recolección de los datos resultantes del CHAEA, y 3 ) el análisis de datos para identificar de manera individual el EA de cada estudiante, y el perfil predominante de toda la muestra. Se espera que, a futuro, los resultados del estudio sirvan de fuente para el desarrollo de planes educativos de mejora y nuevas estrategias pedagógicas por parte de los maestros y la institución en general.

\subsection{Estilos de aprendizaje}

Todos los seres humanos poseen capacidades y habilidades que con el tiempo se estructuran, perfilan, y acentúan, y aunque la educación, en medio de la estandarización del diseño curricular, ha propendido por potencializar estos factores. Lo cierto es que no todos poseen las mismas facultades y potencialidades en el desarrollo de sus habilidades y competencias, que por supuesto no está ligado al desarrollo de la inteligencia, como lo plantea Honey, Munford (1986), pues esta no es tan flexible como lo son los EA. 
Los EA están orientados a la determinación de características propias y particulares de cada uno de los individuos que conforman un grupo social, que además, delimitan de acuerdo a Dunn, Dunn y Price (1985), los métodos para fortalecer el proceso de formación académica, ya que dependiendo de las características particulares, puede desarrollarse una estrategia pedagógica enriquecedora y efectiva que le permita absorber la información. En la concepción de Riechmann (1979, p.2), los EA delimitan los "comportamientos y actitudes relacionados con el contexto de aprendizaje". No obstante, la perfilación de los EA se influencia por diversos factores que hacen parte del contexto social de los grupos humanos. Como se mencionó anteriormente, la familia, constituye un primer acercamiento a la delimitación de las características de cada individuo, pero el vínculo que se establece a partir de la edad, la cultura, entre otros, puede estructurar en algunos individuos, uno o varios EA que pueden de igual forma modificarse, cuando éste cambia de rol o grupo social. Por otro lado, Dunn, et al. (1985), añade, que el aprendizaje depende también de la percepción y las preferencias que tenga el individuo y de cómo este las asuma, es decir, no todos los individuos tienen el mismo interés, y la percepción de ese interés puede variar de persona a persona, aunque el EA sea el mismo. Es este aspecto, sería erróneo decir que hay estilos puros o únicos, de igual manera que no existen estilos de personalidad idénticos. Cada persona usa diferentes EA, pero también suele existir que uno de ellos sea mucho más predominante que los otros y/o se complementen, pero, un estilo no necesariamente es más efectivo que el otro.

A pesar de que existen bastantes clasificaciones de los EA, una de las más usadas es la de Honey y Mumford (1986), que los compila en cuatro estilos:

- Activos: les gustan las cosas nuevas, los temas nuevos, son de mente abierta, no son escépticos y se acomodan fácilmente a actividades o tareas nuevas. Sus principales características son: Animador, Improvisador, Arriesgado y Espontáneo.

- Reflexivos: privilegia la reflexión a la acción y mira las situaciones desde diversos ángulos. Presta atención a las experiencias que vivencia. Sus características predominantes son: Ponderado, Concienzudo, Receptivo, Analítico y Exhaustivo.

- Teóricos: siempre están en búsqueda de la racionalidad y la objetividad. Se alejan de lo subjetivo y lo ambiguo. Las características que tienen son: Metódico, Lógico, Objetivo, Crítico y Estructurado.

- Pragmáticos: son efectivos al momento de tener que ir a la acción. Llevan a cabo asertivamente las actividades, ideas y proyectos que les llama la atención. Sus características son: Experimentador, Práctico, Directo y Eficaz.

La labor del docente, como parte del proceso de enseñanza, es tener en cuenta los intereses de los estudiantes, es decir qué desean alcanzar y cuál es su meta. Desconocer el contexto, limita las buenas prácticas pedagógicas, puesto que, si un estudiante pierde el interés y se desmotiva, puede ocasionar en un momento determinando la dispersión de lo explicado y de esta manera, poco a poco, irá desviándose de su meta personal como profesional. Para el docente conocer el EA de cada estudiante, implica realizar una planeación que será mucho más efectiva, pues estará atrayendo a través de estrategias didácticas la atención de estos, garantizando que lo que enseña, el estudiante sí lo aprenda.

\section{Metodología}

La investigación es de carácter cualitativo, ya que esta permite definir parámetros para abordar un fenómeno desde diferentes conceptualizaciones, entre ellas está el estudio de caso, que es la base sobre la cual se aborda el estudio, y que según Berg (1998) "el método de estudio de casos supone la recolección sistemática de información sobre una persona, ambiente social, evento o grupo, que permite al investigador entender de forma efectiva cómo operan o funcionan", lo cual representa la segunda fase del estudio. Además, es un estudio descriptivo, ya que con base en el análisis de los datos recolectados en el Cuestionario Honey-Alonso Estilos de 
Aprendizaje (CHAEA) se logrará la descripción o identificación del EA predominante de la población objeto de estudio.

\subsection{Muestra}

Para la aplicación del CHAEA se seleccionó una población de 51 estudiantes de la Licenciatura en Lenguas Extranjeras con énfasis en inglés de la UCEVA categorizados en dos grupos. Grupo 1, conformado por 34 estudiantes de primer semestre con una edad promedio de 19 años, y Grupo 2 conformado por 17 de segundo semestre con una edad promedio de 22 años. Esta selección fue aleatoria, es decir, no tuvo en cuenta ninguna característica en especial, buscando evitar sesgos, ya que se buscaba conocer el EA predominante en los semestres iniciales.

Para la segunda fase de recolección y análisis de datos, es preciso detallar que el cuestionario se encuentra sistematizado en hojas de cálculo en el programa EXCEL el cual tiene las fórmulas predeterminadas para arrojar de manera inmediata el resultado del estilo de aprendizaje al estudiante que lo responde, y a su vez empieza a alimentar la estadística en la matriz de resultados en el mismo software a través de números y gráficos. En consecuencia, el muestreo se llevó a cabo en una de las salas de sistemas cada estudiante debió responder los 80 enunciados del CHAEA de carácter dicotómico, donde 1 (uno) representa que esa característica aplica, y 0 (cero) no aplica.

\section{Resultados}

Al terminar la encuesta el estudiante pudo observar su estilo predominante y ver los componentes del mismo, es decir, la descripción del estilo y llevarse una percepción inicial.

Gráfico 1

Resultados Perfil individual de EA predominante

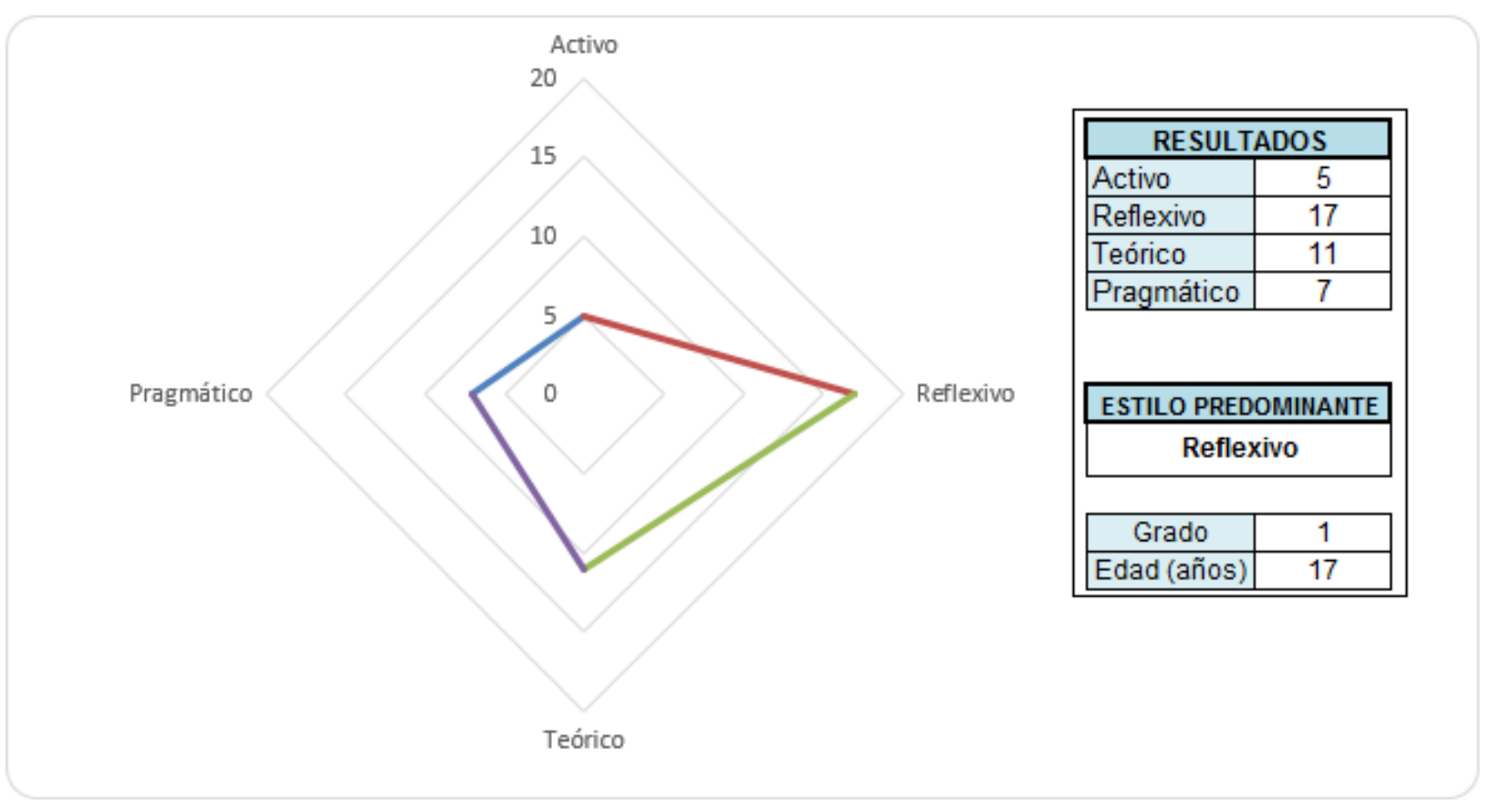

Fuente: Autores

A continuación, se presentan los resultados consolidados por cada grupo. 
Gráfico 2

Histograma de EA Predominante Grupo 1

Estudiantes de I semestre

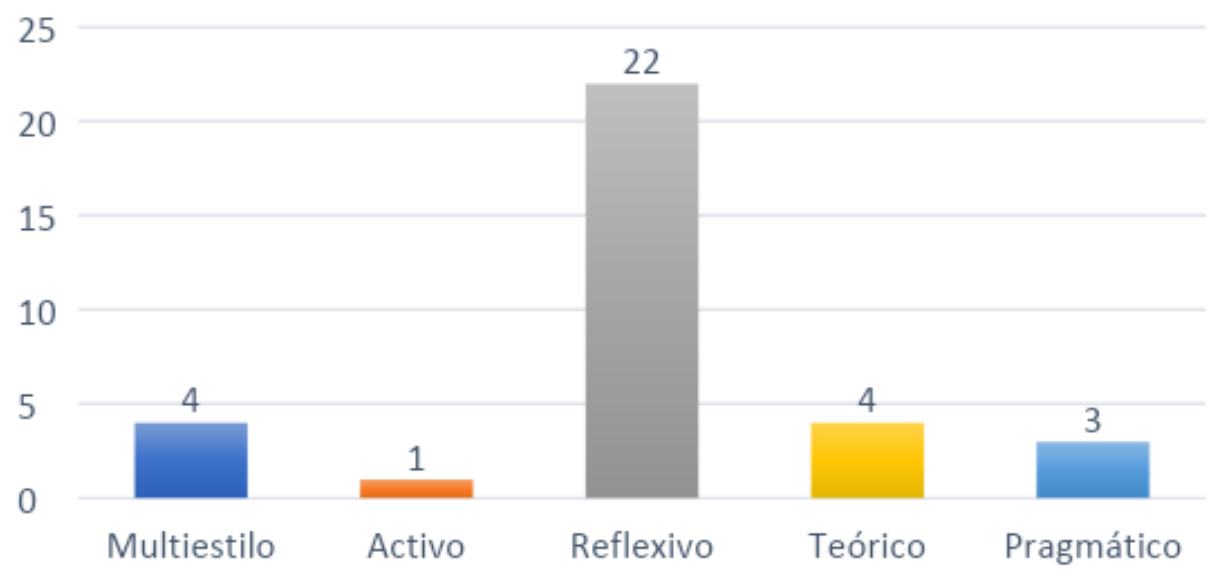

Fuente: Autores

Gráfico 3

Histograma de EA Predominante Grupo 2

Estudiantes de II semestre

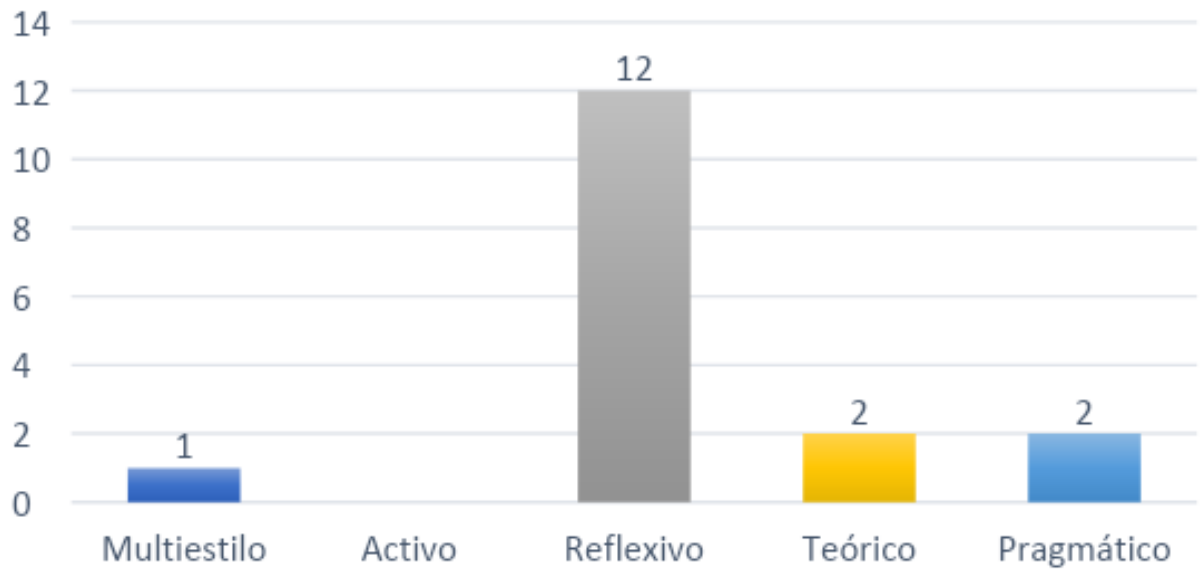

Fuente: Autores

Se evidencia de manera clara que, en los dos grupos evaluados, para 34 estudiantes que representan el $67 \%$ de la población, el EA predominante fue el Reflexivo. En este contexto, los docentes deben incentivar el proceso de aprendizaje mediante actividades pedagógicas que den respuesta al porqué de situaciones del mundo real, en las cuales el estudiante dé a conocer sus conceptos a otros integrantes del grupo con el objetivo de llegar a conclusiones más profundas de los temas analizados (Aragón y Jiménez, 2009). Igualmente, según Hidalgo y Manzano (2009), este tipo de estilo está muy relacionado con las estrategias de aprendizaje que involucren la lectura de la lengua extranjera en los estudiantes, ratificando los resultados obtenidos en el presente estudio.

Asimismo, se observa que no hay diferencias amplias con relación a los otros estilos ubicándose en segundo lugar el estilo Teórico, seguido del Pragmático y el Multiestilo con igual cantidad. Y por último el Activo. Cabe resaltar 
el hecho de que 5 estudiantes que representan el 9.8\% en total tengan un Multiestilo, lo que significa que no tienen un estilo predominante, lo que ratifica Ventura et al. (2014), al afirmar que un estudiante debería estar educado en más de un estilo, aunque ninguno tuviera más peso que otro.

Dichos resultados paralelos en ambos grupos, llevan a estimar que los estudiantes usan varios EA de acuerdo a las necesidades o exigencias de las diversas asignaturas. A esto se debe agregar que depende del tipo de enseñanza que imparten los docentes, las actividades y EA predominantes en la institución. Como ya se mencionó anteriormente, los estudiantes con estilo Reflexivo privilegian la reflexión a la acción, además que observan con detenimiento las diversas experiencias. Estas características a su vez les dan unas ventajas importantes sobre el nivel de abstracción de la información recibida al momento de la comprensión de textos y tareas que surgen del acto lector, y que permiten llegar a conjeturas de ideas generales y específicas de un texto por un análisis acertado teniendo en cuenta la característica del mismo estilo (Pantoja, Duque y Correa, 2013). Por ejemplo, se pueden imaginar o predecir el título de un texto si se le presentan a elegir cuál sería el más apropiado.

Por otro lado, también fue importante identificar los EA predominantes por género. En este caso en el Grupo 1, conformado por 13 hombres (38\%) donde predominó el EA Reflexivo, seguido del Multiestilo, y de 21 mujeres (62\%) donde también predominó el EA Reflexivo, y pocas diferencias entre los demás estilos.

Gráfico 4

EA Predominante por género Grupo 1

Estudiantes de I semestre

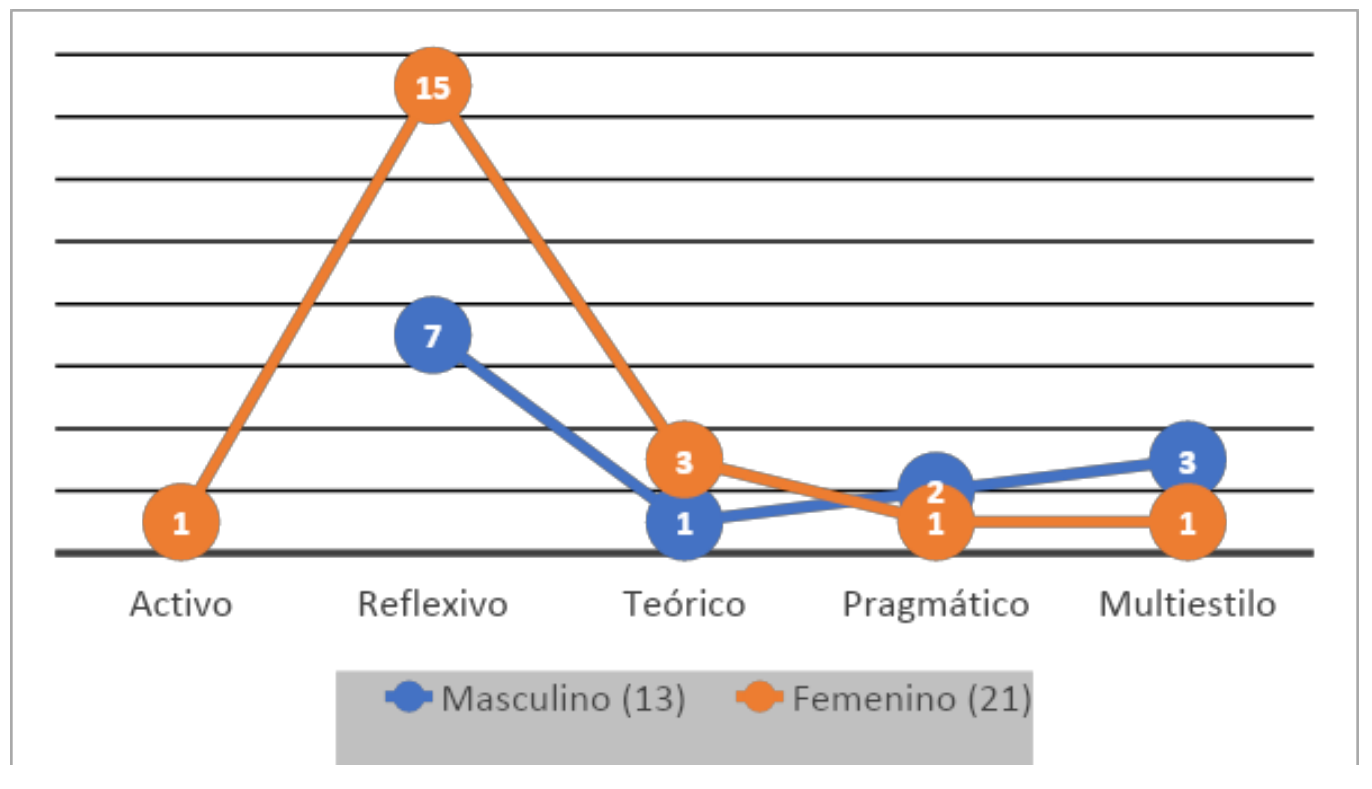

Fuente: Autores

Y para Grupo 2, conformado por 6 hombres (35\%) donde predominó el EA Reflexivo, seguido del Multiestilo, y de 11 mujeres (65\%) donde también predominó el EA Reflexivo, y pocas diferencias entre los demás estilos. 
Gráfico 5

EA Predominante por género Grupo 2

Estudiantes de II semestre

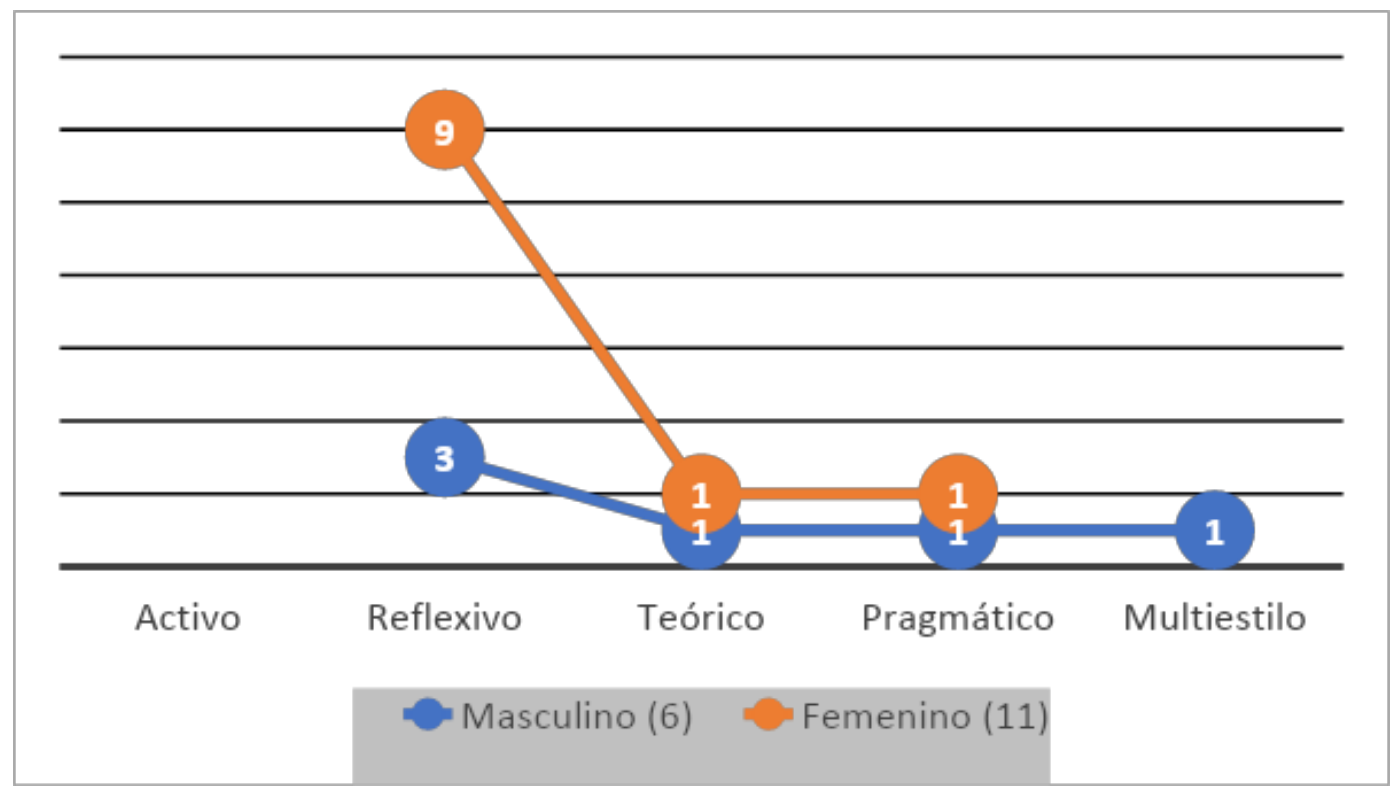

Fuente: Autores

Finalmente es importante enfatizar que no existe una relación muy marcada entre los EA, la edad y género de los estudiantes, donde prácticamente los resultados fueron los mismos en términos de porcentaje.

\section{Discusión}

Enseñar, es un proceso complejo, que no responde simplemente al interés que pueda o no prestar el estudiante en la aprehensión e interiorización del conocimiento, sino que responde a una serie de factores que determinan cómo, qué y cuando el estudiante aprende, dependiendo de su EA predominante. En el actual escenario de esta investigación resulta importante mencionar que el estilo Reflexivo, se logra siempre y cuando el estudiante pueda realizar una observación de la situación, deliberar acerca de lo que acontece en una situación dada, tomar decisiones coherentes y lógicas para recolectar información, analizar las posibles causas y consecuencias antes de actuar.

Por otro lado, según Coffield et al. (2004), una aplicación pertinente de instrumentos para medir los EA provee diferentes ventajas tanto para docentes como para estudiantes, en la medida que a través del conocimiento de habilidades, fortalezas y debilidades, se pueden diseñar estrategias que permitan fortalecer el aprendizaje, y el estudiante crea una consciencia sobre su propio aprendizaje, lo cual beneficia ambas partes en el alcance de objetivos sobre el conocimiento en las diferentes áreas y competencias.

Tales las estrategias pedagógicas requieren de una innovación continua para garantizar la efectividad de la misma. Si el maestro quiere dinamizar los procesos de formación académica en la escuela, debe fortalecer la manera de enseñar. En el caso de la lectura, estas estrategias deben atacar el interés individual de cada estudiante, y repercutir en la comprensión sobre lo que él lee y lo que el autor desea transmitir, de lo contrario, el mensaje no será recibido ni comprendido, resultando en una desmotivación por el aprendizaje. (Cassany, 2004; Cabrales, Oliveros y Mejía, 2014; Loaiza y Galindo, 2014). En el caso de la enseñanza del inglés es menester de los de los sistemas educativos y docentes contribuir al desarrollo de competencias lectoras en sus estudiantes, a fin de mejorar los resultados de las pruebas estandarizadas posicionando al bilingüismo como uno de los 
elementos más fuertes en el campo educativo, pero para ello, se requiere cambios drásticos en la forma cómo se enseña esta área.

Ahora bien, los EA en el aula sugieren entonces, romper con esquemas tradicionales en el proceso de enseñanza. Para el caso del desarrollo de la competencia lectora en inglés y de acuerdo a los EA encontrados, es necesario reflexionar sobre la enseñanza de esta área, enfocándose en incorporar estrategias innovadoras que relacionen los elementos base de los EA y los actuales recursos educativos digitales (Contreras y Eguia, 2016; Corchuelo, 2018), pues de acuerdo a Niño (2016), las tecnologías ya se han adentrado al campo educativo, y es necesario apropiarse e incorporarlas al contexto educativo. Sin embargo, éstas deben resultar atractivas, sin perder su intencionalidad, que es contribuir, en este caso, a la enseñanza del inglés.

\section{Conclusiones}

Con el análisis realizado sobre los EA y la identificación del más predominante, es necesario entender la diversidad que puede tener un estudiante con relación a sus costumbres, conocimientos, capacidades y aptitudes, para de esta manera generar estrategias y didácticas innovadoras que integren los saberes cognitivos con metodologías pedagógicas que contribuyan al desarrollo de habilidades de lectura, iniciando con los futuros docentes de inglés como lengua extranjera.

Asimismo, es vital reconocer que los EA evolucionan paulatinamente y se van perfeccionando con el tiempo. Por tanto, es erróneo pensar que tal desarrollo sólo sea tarea de la educación básica primaria, secundaria y media, pues, en muchos casos, los EA se vislumbran, aún más en el campo universitario, donde el nivel de conciencia del estudiante está más desarrollado a la vez que es consciente y coherente de sus actos.

Es por ello que se hace imprescindible que el maestro, detecte y conozca cuáles es el EA de sus estudiantes, de tal forma que le permita usarlos como herramientas que hagan la lectura se enseñe en contexto, y le facilite diseñar estrategias que garanticen el aprendizaje de lo que se enseña, permitiendo que este sea significativo y aplicado en su entorno real. Por supuesto que resulta más complejo para el docente diseñar actividades para diversos EA, pero, si se quiere lograr un aprendizaje verdaderamente significativo, es necesario que este se involucre en el diseño curricular de las asignaturas, y realizar un seguimiento y evaluación continua que permita verificar en qué momento, los EA se enfocan en el estudiante de forma clara (García-Retana, 2013). Empero, es claro que la diversidad de EA, estará presente en todos los grupos, pero, entre ellos, habrá uno que sobresalga.

En este contexto, existe una relación significativa entre el aprendizaje, los EA, la enseñanza y el desarrollo de la competencia lectora, lo que valida la integración de una estrategia pedagógica que involucre elementos tecnológicos en el aprendizaje, ya que esto resulta atractivo y en la mayoría de los casos de más fácil comprensión y adaptación para el estudiante digital; de forma tal que se involucren adelantos investigativos en asuntos relacionados como la estrategias de Gamificación, dispositivos web o móviles (Sandoval et al, 2020), el uso de redes sociales (Salazar et al. 2019), entre otros.

Finalmente, se espera que los resultados obtenidos en la investigación sean de ayuda y alimento para la formulación de nuevas estrategias pedagógicas, tanto en los docentes, como en la misma institución, con el objetivo de mantener actualizados los currículos y brindar mejores condiciones a los estudiantes, de tal forma que el plan académico resulte atractivo e innovador, marcando diferencias que lo posicionan como uno de los mejores de la región, y sea reconocido por su alta calidad académica.

\section{Referencias bibliográficas}

Alonso, C., Gallego, D. y Honey, P. (1994). Los Estilos de Aprendizaje. Bilbao. España. 
Alonso, C., Gallego, D. y Honey, P. (2007). Los estilos de aprendizaje. Procedimientos de diagnóstico y mejora (2a ed.). Bilbao: Ediciones Mensajero. España.

Aragón, M. y Jiménez, Y. (2009). Diagnóstico de los estilos de aprendizaje en los estudiantes: Estrategia docente para elevar la calidad educativa. CPU-e, Revista de Investigación Educativa, 9. Recuperado de http://www.uv.mx/cpue/num8/opinion/aragon_estilos_aprendizaje.html

Berg, B. (1998) "Qualitative Research Methods for the Social Sciences". Allyn \& Bacon, Needham Heights.

Cabrales, M., Oliveros, M. y Mejía, A. (2014). Estilos de aprendizaje en estudiantes de inglés y su rendimiento académico. Itinerario Educativo, XXVIII (63), 155-177. DOI: https://doi.org/10.21500/01212753.1484

Cassany D. (2004). Explorando las necesidades actuales de comprensión: aproximaciones a la comprensión crítica. Lectura y vida. (2), 6-23. Recuperado de https://repositori.upf.edu/handle/10230/21237

CERLALC (2012). El libro en cifras. Boletín estadístico del libro en Latinoamérica. Recuperado de https://cerlalc.org/wp-content/uploads/publicaciones/olb/PUBLICACIONES_OLB_El-libro-en-cifras1_v1_010812.pdf

Coffield, F., Moseley, D., Hall, E. y Ecclestone, K. (2004). Should we be using learning styles? What research has to say to practice. London: Learning and Skills Research Centre, University of Newcastle

Corchuelo, C. (2018). Gamificación en educación superior: experiencia innovadora para motivar estudiantes y dinamizar contenidos en el aula. Edutec. Revista Electrónica De Tecnología Educativa, (63), 29-41. https://doi.org/10.21556/edutec.2018.63.927

Contreras, R. y Eguia, J. (2016). Gamificación en aulas universitarias. Bellaterra : Institut de la Comunicació, Universitat Autònoma de Barcelona. Recuperado de https://ddd.uab.cat/record/166455

De la Fuente, J. y Justicia, F. (2003). Regulación de la enseñanza para la autorregulación del aprendizaje en la universidad. Aula Abierta, 82, 161-171. https://dialnet.unirioja.es/servlet/articulo?codigo=1012077

Dunn, R., Dunn, K. y Price, G. (1985). Manual: Learning Style Inventory. Lawrence,Kansas: Price Systems.

El Espectador. (2014). Colombianos disminuyen hábito de lectura durante 2014, dice estudio. El Espectador. Recuperado de https://www.elespectador.com/noticias/nacional/colombianos-disminuyen-habito-delectura-durante-2014-d-articulo-509326

El País. (2013). Superar el nivel básico de inglés en los colegios, el reto para Cali y el Valle. El País. Recuperado de https://www.elpais.com.co/cali/superar-el-nivel-basico-de-ingles-en-los-colegios-el-reto-para-y-elvalle.html

El Tiempo. (2018). Colombia está todavía muy lejos de ser bilingüe. El Tiempo. Recuperado de https://www.eltiempo.com/vida/educacion/bilingueismo-en-colombia-no-avanza-42776

García-Retana, J. (2013). Reflections on learning styles and calculus learning in engineering majors. Investig. en Educ., 13(1), 362-390. Recuperado de http://www.scielo.sa.cr/scielo.php?script=sci_arttext\&pid=S1409$47032013000100014 \& \operatorname{lng}=e n \&$ tIng=

Grabe, W., y Stoller, F. L. (2002). Teaching and Researching Reading. Great Britain: Pearson Education. http://www.lecturayvida.fahce.unlp.edu.ar/numeros/a25n2/25_02_Cassany.pdf

Honey, P. y Mumford, A. (1986) Using our learning styles. Berkshire, U.K.: Peter Honey 
Loaiza, N. y Galindo, A. (2014). Estilos de aprendizaje de segundas lenguas y formación bilingüe consecutiva en educación primaria, secundaria y superior: hacia un estado del arte. Lenguaje, 42 (2), 291-314.

Recuperado de http://www.scielo.org.co/pdf/leng/v42n2/v42n2a04.pdf

Loayza, R. (2017). Estilos de aprendizaje y comprensión lectora en estudiantes de 4to. grado del colegio Santa Rosa, Huacho - 2017. Tesis de maestría. Escuela de posgrado, Universidad César Vallejo, Perú. Recuperado de http://repositorio.ucv.edu.pe/handle/20.500.12692/21706

Hidalgo, D. y Manzano, D. (2009). Estilos de aprendizaje, estrategias de lectura y su relación con el rendimiento académico de la lengua extranjera. Revista Educación, 21(12), 123-150. Recuperado de http://redalyc.uaemex.mx/redalyc/src/inicio/ArtPdfRed.jsp?iCve=70611919007

Monereo, C. (2002). Estrategias de aprendizaje, 2ª ed., España: Antonio Machado Libros.

Monereo, C. (2007). Estrategias de enseñanza y aprendizaje. Formación del profesorado y aplicación en la escuela (10a ed.). España: Grao.

Pantoja, M., Duque, L. y Correa, J. (2013). Modelos de estilos de aprendizaje: una actualización para su revisión y análisis, Revista Colombiana de Educación, 64. http://www.scielo.org.co/pdf/rcde/n64/n64a04.pdf

Paul, R. y Elder, L. (2005). Estándares de Competencias para el Pensamiento Crítico. Estándares, principios, desempeño, indicadoes y resultados con una rúbrica maestra en el pensamiento crítico. Recuperado de https://www.criticalthinking.org/resources/PDF/SP-Comp_Standards.pdf

Quintana, J., Restrepo, D., Romero, G. y Cárdenas G. (2019). The effect of Content and Language Integrated Learning on the development of English reading comprehension skills. Revista Lenguaje. 47(2). DOI: 10.25100/lenguaje.v47i2.7699

Riechmann, S. (1979). Learning Styles. Their Role in Teaching Evaluation and Course Design. Ann Arbor, Michigan: ERIC Ed

Niño, L. (2016). La motivación hacia el aprendizaje y su relación con las tecnologías de la información y la comunicación. Libros Universidad Nacional Abierta Y a Distancia, 75-82. Recuperado de https://hemeroteca.unad.edu.co/index.php/book/article/view/2607

Sandoval, E., Lasso, L., B, A. y Maldonado, J. (2020). Integration of Gamification for physical fitness inside the classroom through mobile and web technologies. Revista Espacios. 41(20), 185-201. Recuperado de: http://www.revistaespacios.com/a20v41n20/20412015.html

Salazar, A., Pérez, G., González, D., y Romero, G. (2019). Analysis of Closed Facebook Communities to Enhance Vocabulary Development in English as a Foreign Language through Flipped Classroom Method. Revista Red Iberoamericana de Pedagogía REDIPE. 8(8), 168-79. DOI https://doi.org/10.36260/rbr.v8i8.809

Sánchez-Jabba, A. (2013). Bilingüismo en Colombia. Documentos de trabajo sobre economía regional, (191). Recuperado de http://banrep.gov.co/sites/default/files/publicaciones/archivos/dtser_191.pdf

Solé, I. (2012). Competencia lectora y aprendizaje. Revista Iberoamericana de Educación. 59, 43-61. https://doi.org/10.35362/rie590456

Ventura, A., Palou, I., Széliga, C. y Angelone, L. (2014). Estilos de aprendizaje y enseñanza en ingeniería: una propuesta de educación adaptativa para primer año. Revista Educación En Ingeniería, 9(18), 178-189. https://doi.org/10.26507/rei.v9n18.461

Esta obra está bajo una Licencia Creative Commons Attribución-NoCommercial 4.0 International

(c) BY-NC 